\title{
DESAFÍOS Y OPORTUNIDADES DEL SISTEMA EDUCATIVO ANTE LA INMIGRACIÓN Y LA DIVERSIDAD CULTURAL
}

\author{
CHALLENGES AND OPPORTUNITIES OF THE EDUCATIONAL SYSTEM \\ IN THE FACE OF IMMIGRATION AND CULTURAL DIVERSITY
}

\section{DESAFIOS E OPORTUNIDADES DO SISTEMA EDUCACIONAL DIANTE DA IMIGRAÇÃO E DA DIVERSIDADE CULTURAL}

Pedro Garrido Rodríguez ${ }^{1}$

\begin{abstract}
RESUMEN
Este artículo nace de la participación en el II Coloquio Internacional Educación e Interculturalidad, celebrado en abril de 2019 en la Facultad de Educación de la Universidad de Salamanca. Aborda tres cuestiones clave en nuestro sistema educativo actual. La primera son las variables que intervienen en el proceso de migración con respecto a la educación del alumnado inmigrante. Proceso que depende de factores muy diversos que exceden lo educativo, como el acceso a los servicios fundamentales del estado del bienestar en igualdad de condiciones - las características socioeconómicas de la familia. En segundo lugar, se analiza la situación del alumnado inmigrante en la actualidad, marcada por la baja escolarización en las etapas postobligatorias, un índice de fracaso escolar elevado y expectativas socioeconómicas reducidas. Algo que nuestro sistema educativo debe solventar inexorablemente en cumplimiento con su función de posibilitar la integración, el progreso y el ascenso social. Esto nos lleva a la tercera cuestión: la respuesta que deben ofrecer los sistemas educativos al alumnado inmigrante. Esta ha de pasar siempre por fomentar en todo momento un modelo intercultural, en lo educativo y en lo social. Es decir, un modelo que ofrezca soluciones a las necesidades específicas de los alumnos que lo componen, que posibilite la igualdad de oportunidades y que garantice una educación normalizada, inclusiva y de calidad, generando la interacción y el enriquecimiento mutuo de su alumnado a través de metodologías activas y aprendizaje cooperativo.
\end{abstract}

PALABRAS CLAVE: Inmigración. Diversidad cultural. Educación. Interculturalidad. Educación Intercultural. Sistema Educativo.

\footnotetext{
${ }^{1}$ Doctor por la Universidad de Salamanca, es docente de los Programas de Posdoctorado de Derechos Humanos DHPCBE y GDHDS de la Universidad de Salamanca y de los Programas de Posdoctorado EIPPPD y EIPPS de la Universidade Portuçalense, Oporto. Es miembro investigador del Seminario Internacional de Historia Contemporánea de los Derechos Humanos de la Universidad de Salamanca, del Instituto Jurídico Portuçalense, de la Universidade Portuçalense Infante Dom Henrique (Oporto), del Instituto Complutense de Estudios Jurídicos y Críticos de la Universidad Complutense de Madrid.E-mail: pegarro@usal.es
} Submetido em: -21/01/2021 Aceito em: 08/06/2021 


\section{ABSTRACT}

This article arises from the participation in the II International Colloquium Education and Interculturality, held in April 2019 at the Faculty of Education of the University of Salamanca. It studies three key issues in our current educational system. The first are the variables of the migration process with respect to the education of immigrant students. A process that depends on very diverse aspects that go beyond education, such as access to the fundamental services of the welfare state under equal conditions or the socioeconomic characteristics of the family. Second, the current situation of immigrant students, influenced by low school enrollment in the postcompulsory stages, a high school failure rate and low socioeconomic expectations. Something that our educational system must inexorably solve in compliance with its function of enabling integration, progress and social advancement. This brings us to the third question: the answer that educational systems should offer to immigrant students. This must always go through fostering an intercultural model at all times, in education and in society. In other words, a model that offers solutions to the specific needs of the students that comprise it, that enables equal opportunities and that guarantees a standardized, inclusive and quality education, generating interaction and mutual enrichment of its students through active methodologies and cooperative learning.

KEYWORDS: Immigration. Cultural diversity. Education. Interculturality. Intercultural Education. Educational system.

\section{RESUMO}

Este artigo surge da participação no II Colóquio Internacional de Educação e Interculturalidade, realizado em abril de 2019 na Faculdade de Educação da Universidade de Salamanca. Ele aborda três questões-chave em nosso sistema educacional atual. As primeiras são as variáveis que intervêm no processo de migração no que diz respeito à educação dos alunos imigrantes. Um processo que depende de fatores muito diversos que vão além da educação, como o acesso aos serviços fundamentais do Estado de bem-estar em igualdade de condições ou as características socioeconômicas da família. Em segundo lugar, analisa-se a situação atual dos alunos imigrantes, marcada por uma baixa escolaridade nas fases pós-obrigatórias, uma taxa de repetência do ensino médio e baixas expectativas socioeconômicas. Algo que nosso sistema educacional deve inexoravelmente resolver em cumprimento a sua função de possibilitar a integração, o progresso e a promoção social. Isso nos leva à terceira questão: a resposta que os sistemas educacionais devem oferecer aos alunos imigrantes. Isso deve passar sempre pela promoção de um modelo intercultural em todos os momentos, na educação e na sociedade. Ou seja, um modelo que ofereça soluções às necessidades específicas dos alunos que o compõem, que possibilite a igualdade de oportunidades e que garanta uma educação padronizada, inclusiva e de qualidade, gerando interação e enriquecimento mútuo dos seus alunos através das metodologias ativas e aprendizagem cooperativa.

PALAVRAS-CHAVE: Imigração. Diversidade cultural. Educação. Interculturalidade. Educação intercultural. Sistema educativo. 


\section{INTRODUCCIÓN}

Como queda explícito en el resumen, este artículo pretende analizar cómo afecta la inmigración y la diversidad cultural al sistema educativo y cómo reacciona este ante el crecimiento de aquellas. Las metodologías empleadas son el método histórico de interacción de variables y el análisis crítico del discurso. Básicamente, el primero consiste en analizar el mayor número de variables que intervienen en el objeto de estudio e integrarlas, con objeto de lograr una visión global, panorámica y de conjunto. En cuanto al segundo, consiste en analizar críticamente los elementos que intervienen en la producción de un discurso, especialmente: el agente emisor, el destinatario, los elementos de contexto y la intención comunicativa, esto es, qué pretende conseguir el agente emisor con la producción de su acto de habla. Todo pivota sobre tres cuestiones muy concretas que se irán desgranando a lo largo del artículo y cuya respuesta revela las investigaciones del autor en el ámbito de las migraciones, la diversidad cultural y la educación: las múltiples variables intervinientes en la educación del alumnado inmigrante, la situación actual del alumnado inmigrante en nuestro sistema educativo y la respuesta que este le ha de ofrecer. Estas tres cuestiones se irán desarrollando en el artículo una a una. Su estudio exhaustivo nos lleva finalmente a abogar por el modelo educativo intercultural por ser el que mejor satisface las necesidades de su heterogéneo alumnado, el más coherente con el modelo de sociedad actual y el más competente para cumplir con las garantías previstas por el discurso positivo internacional de Derechos Humanos, paradigma legitimado por la vía del consenso internacional y consolidado mundialmente.

\section{VALORACIÓN GENERAL DEL PROCESO DE LAS MIGRACIONES EN RELACIÓN CON LA EDUCACIÓN DEL ALUMNADO INMIGRANTE. ¿ES SÓLO UNA CUESTIÓN DE LA EDUCACIÓN O ENTRAN OTROS FACTORES?}

Pérez Esparrels y Rahona López (PÉREZ ESPARRELS, RAHONA LÓPEZ, 2009) señalan una serie de factores que son clave en la integración del alumno en la escuela: conocimiento del idioma, la edad de incorporación al sistema educativo, el desfase curricular, las características familiares, el nivel cultural y socioeconómico de la familia, la situación jurídica de la familia, la participación de la familia en la escuela. Algunos de estos factores son eminentemente educativos, pero otros tienen más que ver con las características socioeconómicas y culturales del entorno del alumno. Todos ellos son cruciales para la buena integración y progreso del alumno en la escuela.

A nuestro juicio, es una cuestión que excede, con mucho, la cuestión educativa. Es una cuestión de integración y, por tanto, solo podemos ponderarla tomando en consideración todos los aspectos relacionados con la integración. Actualmente se reconoce el derecho de los alumnos a ser educados en condiciones de igualdad. Sin embargo, esas condiciones de 
igualdad solamente son posibles si partimos de una integración educativa y social real. Para lograrla, por tanto, son necesarias medidas educativas y también sociales. La Comisión Europea establece en sus Principios Básicos Comunes, dentro del marco del Programa Europeo de La Haya (2005-2010), que la integración es un proceso bidireccional. Por tanto, hay una cuota de responsabilidad tanto en la persona que se integra como en la sociedad de acogida. Es preciso desarrollar con más profundidad la cuestión de los Principios Básicos Comunes del Programa de La Haya porque son de inmenso interés para dentro del tema que estamos tratando. El Programa propone para cada Principio Básico Común actuaciones específicas a realizar tanto a nivel de la Unión Europea como a nivel nacional. Es de vital importancia constatar que la integración es fundamental en la educación y, al contrario, la educación integral, inclusiva y de calidad es fundamental también para la integración.

Principio Básico Común 1. "La integración es un proceso bidireccional y dinámico de ajuste mutuo por parte de todos los inmigrantes y residentes de los Estados miembros".

Acciones a nivel de la UE: financiar acciones transnacionales, como campañas o manifestaciones interculturales destinadas a difundir información precisa y exacta sobre las culturas, las religiones y las contribuciones económicas y sociales de los inmigrantes y financiar proyectos piloto y estudios destinados a explorar nuevas formas de cooperación comunitaria en el ámbito de la admisión y la integración. Acciones a nivel nacional: reforzar la capacidad de la sociedad de acogida para adaptarse a la diversidad a través de medidas de fomento de la integración dirigidas a la población de acogida; elaborar programas nacionales para la aplicación de un enfoque bidireccional; mejorar la comprensión y aceptación de la inmigración mediante campañas de sensibilización, exposiciones, manifestaciones interculturales, etc.; promover en la sociedad de acogida el conocimiento de las consecuencias de los programas de acogida y admisión; reforzar el papel del sector privado en la gestión de la diversidad; promover la confianza y las buenas relaciones en los barrios, por ejemplo mediante iniciativas de bienvenida, acciones de "padrinazgo", etc.; y cooperar con los medios de comunicación, por ejemplo mediante el establecimiento de códigos de conducta facultativos para los periodistas.

Principio Básico Común 4. "Un conocimiento básico del idioma, la Historia y las instituciones de la sociedad de acogida es indispensable para la integración; permitir a los inmigrantes adquirir ese conocimiento básico es esencial para que la integración tenga éxito".

Acciones a nivel de la UE: financiar programas o modelos de integración innovadores que incluyan una formación lingüística y talleres de comunicación, así como información sobre los aspectos culturales, políticos y sociales del país de acogida. Acciones a nivel nacional: reforzar el aspecto de integración de los procedimientos de admisión mediante, por ejemplo, medidas preparatorias en el país de origen que incluyan la distribución de paquetes informativos y la organización de cursos de lenguas y educación cívica en dicho país de origen; organizar programas y actividades de acogida para los nacionales de terceros países recién

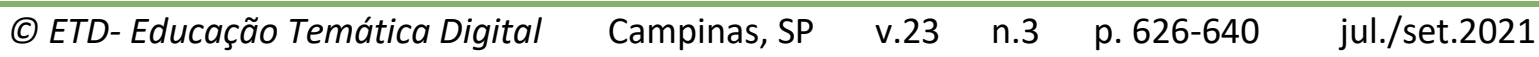


llegados, al objeto de que adquieran unos conocimientos básicos sobre la lengua, la historia, las instituciones, las características socioeconómicas, la vida cultural y los valores fundamentales del país de acogida; proponer cursos de distintos niveles teniendo en cuenta la formación de los interesados y sus conocimientos previos sobre el país; reforzar la capacidad de los programas y actividades de acogida para las personas a cargo de los inmigrantes sujetos a un procedimiento de admisión mujeres, niños, ancianos, analfabetos y minusválidos; incrementar la flexibilidad de los programas de acogida mediante cursos de media jornada y nocturnos, módulos acelerados y cursos a distancia y en línea; orientar las actividades de acogida hacia los jóvenes nacionales de terceros países con problemas sociales y culturales específicos vinculados a problemas de identidad, a través, en particular, de programas basados en los conceptos de tutoría o transmisión de modelos; y agrupar los recursos para permitir a los municipios vecinos ofrecer distintos tipos de cursos.

Principio Básico Común 5. "Los esfuerzos realizados en la educación son fundamentales para preparar a los inmigrantes, y en particular a sus descendientes, a participar con más éxito y de manera más activa en la sociedad".

Acciones a nivel de la UE: incorporar los objetivos de la integración en los distintos programas de la Comisión relacionados con la educación; promover la educación de los nacionales de terceros países a través del programa de trabajo titulado «Educación y formación 2010»; y promover un reconocimiento transparente de las cualificaciones, especialmente mediante propuestas para la creación de un marco europeo de cualificaciones. Acciones a nivel nacional: reflejar la diversidad en los planes educativos; tener en cuenta los problemas específicos de los jóvenes inmigrantes a través de medidas destinadas a prevenir el fracaso escolar; mejorar el acceso de los jóvenes inmigrantes a la enseñanza superior; y combatir eficazmente la delincuencia entre los jóvenes inmigrantes.

Principio Básico Común 8. "La práctica de diversas culturas y religiones está garantizada por la Carta de los Derechos Fundamentales y debe quedar salvaguardada, a menos que dichas prácticas entren en conflicto con otros derechos europeos inviolables o con la legislación nacional".

Acciones a nivel de la UE: facilitar un diálogo intercultural e interconfesional a nivel europeo que incluya a las distintas partes interesadas y desarrollar ulteriormente el diálogo de la Comisión con organizaciones humanistas y religiosas. Acciones a nivel nacional: desarrollar un diálogo intercultural constructivo, estimulando una reflexión pública en profundidad y promover plataformas de diálogo intra e interconfesionales entre las comunidades religiosas y/o entre las comunidades y los responsables políticos. 
Es importante enfatizar que no se trata solamente de un trabajo educativo, es necesario integrar y es necesario concienciar para que la sociedad de acogida tenga una actitud proactiva con el principio de la bidireccionalidad de la integración y sea proclive a la interculturalidad. Sin embargo, las estadísticas más recientes nos muestran como las opiniones sociales con respecto a la diversidad cultural y a la interculturalidad son ambivalentes. Conviven los discursos reactivo y proactivo.

\section{SITUACIÓN ACTUAL DE LA ESCOLARIZACIÓN DEL ALUMNADO INMIGRANTE EN ESPAÑA DESDE EN PLAN LEGAL Y PEDAGÓGICO}

El alumnado inmigrante presenta una serie de carencias y necesidades que se mantienen a lo largo del tiempo y que hoy en día no se han logrado solucionar. Nos referimos principalmente al alto índice de fracaso escolar, falta de integración efectiva, baja escolarización en las etapas postobligatorias y pocas expectativas socioeconómicas. En este sentido, la educación no está cumpliendo con su función de posibilitar el progreso y el ascenso social y hay una serie de factores que lo explican.

Las limitaciones presupuestarias dificultan la dotación de recursos suficientes. El porcentaje del PIB destinado a la educación es inferior en varios puntos a la media comunitaria. La LOE (2006) preveía equiparar progresivamente el gasto a los niveles de la UE en los siguientes 10 años y aumentar así los recursos para desarrollar políticas compensatorias en las áreas que precisan atención especial: "Art. 155.2: El Estado y las Comunidades Autónomas acordarán un plan de incremento del gasto público en educación para los próximos diez años, que permita el cumplimiento de los objetivos establecidos en la presente Ley y la equiparación progresiva a la media de los países de la Unión Europea".

Existen desigualdades en el acceso y en el desarrollo escolar del alumnado inmigrante. Por un lado, se tiende a escolarizar a los alumnos inmigrantes mayoritariamente en los centros públicos, los cuales concentran y segregan a este colectivo. Por otro lado, hay una gran tasa de absentismo y de fracaso escolar. Este fracaso escolar se vincula especialmente a un contexto de exclusión social que sufren muchas familias inmigrantes, como atestigua el Informe PISA, realizado por la OCDE (Organización para la Cooperación y el Desarrollo Económico).

Hay importantes carencias y necesidades que se mantienen en el tiempo. Por múltiples causas como haber sido escolarizados previamente en condiciones distintas a las de la sociedad de acogida, haber sido objeto de escolarización interrumpida o irregular, haber sufrido en el país de origen exclusión por pertenencia a una etnia o grupo social o por presentar alguna necesidad educativa especial a la que no se le ha sabido dar respuesta. Hay algunas necesidades educativas especiales frecuentes en el alumnado inmigrante: desconocimiento de la cultura, las tradiciones, las normas y los hábitos de la comunidad educativa y de la sociedad de acogida y la carencia de las competencias lingüísticas, 
especialmente los alumnos que no proceden de países hispanohablantes. Todo ello requiere medidas de flexibilización curriculares y organizativas, contemplando la necesaria contextualización de los centros y de sus necesidades socioeducativas, incidiendo especialmente en una escolarización lo más temprana posible.

Uno de los grandes retos del sistema educativo español es mejorar la formación inicial y continua de los profesores, de forma que adquieran conocimientos suficientes para responder a los cambios de la sociedad y a las necesidades específicas del alumnado inmigrante. Para ello, se deben generalizar dinámicas de formación-reflexión-acción, incorporando nuevos marcos teóricos y buenas prácticas para gestionar la diversidad cultural. Esto parte de la premisa de unas altas expectativas por parte del profesorado hacia los alumnos inmigrantes. Hay que eliminar los prejuicios de las diferencias sociales y culturales y buscar un conocimiento objetivo de ellas. Han de buscarse asociaciones entre educación formal e informal, investigación y práctica y ampliar el espectro de las acciones educativas.

Existen factores que dificultan la implicación familiar, como la precariedad económica de las familias, la vulnerabilidad por exclusión social, la falta de tiempo, la divergencia de referentes culturales, la ausencia de formación o experiencia académica. Para contrarrestar estas dificultades, es preciso incluir actuaciones de formación y sensibilización que incorporen a las familias a la vida escolar, tanto en la acogida como a lo largo de todo el proceso educativo. Estas actuaciones deben contemplar actividades de igualdad de oportunidades entre niños y niñas. También debe aprovecharse en este mismo sentido la acción tutorial de los profesores y las AMPA con las familias autóctonas e inmigrantes y la educación en valores como eje transversal en todas las materias de la escuela.

En lo relativo a la educación en valores, ciudadanía e integración, en los últimos años, está aconteciendo un crecimiento preocupante de actitudes sociales intolerantes, racistas y xenófobas entre la juventud española. Existen ciertos problemas que complican la integración, como la falta de mediadores específicos para las diferentes culturas, la baja participación de las familias inmigrantes en las AMPA, la necesidad de programas de acogida para los recién llegados y de servicios de apoyo y asesoramiento a las familias y a los centros o la escasez de acciones complementarias en necesidades no estrictamente curriculares. El sistema educativo debe responder a estas necesidades, como se apunta desde la sociología de la educación. En este sentido, la formación en valores y para la ciudadanía tienen un importante papel que cumplir.

La formación continua es clave. Sin embargo, los datos indican que esta se promueve poco entre los inmigrantes. Su baja tasa de escolarización no obligatoria puede causar problemas de integración social a medio y largo plazo. Se necesitan programas de formación profesional continua en colaboración con empresas que den opciones de futuro a los jóvenes y los integren en el sistema productivo. Los programas de cualificación profesional inicial para el alumnado mayor de 16 años van en esta línea. También es fundamental la formación

$\begin{array}{llllll}\text { (c) ETD-Educação Temática Digital } & \text { Campinas, SP } & \text { v.23 } & \text { n.3 } & \text { p.626-640 } & \text { jul./set.2021 }\end{array}$


permanente, de cara al empleo y también de cara a desenvolverse en una sociedad cambiante adquiriendo nuevas competencias, como sostiene la UE. Es preciso mejorar la oferta de programas de formación para adultos inmigrantes. Éstos deben incluir en una primera fase el aprendizaje de la lengua y de los aspectos más esenciales de la sociedad de acogida; además de la cualificación profesional y el aprendizaje permanente. Para ello, se debe incorporar la perspectiva de la diversidad cultural en todos los procesos educativos, con adecuación de la formación del profesorado a las características de su alumnado. A la vez, hay que sensibilizar a la población inmigrante adulta sobre el vínculo entre promoción sociolaboral y formación continua, instaurando así actitudes positivas hacia este aprendizaje continuo.

La variable de género debe estar presente. Un modelo de Educación Intercultural efectivo debe incorporar también, como se afirma en el Informe sobre la Integración del Alumnado Extranjero en la Escuela en 2008, la variable de género. En este momento, existen en el ámbito educativo español algunas carencias que el Plan Estratégico de Igualdad de Oportunidades (2007-2011) pone de manifiesto: 1) Matriculación diferencial. Más del 54\% del alumnado universitario es femenino, en contraposición a la antigua falta de matriculación femenina; 2) Segregación vertical y horizontal del profesorado. Si revisamos los datos, es palpable la diversificación del profesorado por etapas educativas: la proporción de mujeres en el total del profesorado en todos los niveles es del 65\%. Sin embargo, los porcentajes varían mucho dependiendo del nivel concreto. Así, el porcentaje de mujeres en Educación Infantil y Primaria es del $77 \%$, mientras que en la Universidad baja al $35 \%$ y hasta sólo el $14 \%$ en el cargo de catedráticas; 3) Falta de plazas para la escolarización de 0-3 años, que afecta más las mujeres jóvenes para corresponsabilizar tareas y las lleva a tener que decidir entre la maternidad y la carrera profesional; 4) Escasez de mujeres ocupando cargos directivos o influyentes en la toma de decisiones, la cual es todavía una realidad en todos los ámbitos del mercado de trabajo; 5) Carencia de análisis cualitativos y cuantitativos de la práctica educativa, los libros de texto y demás material educativo y los contenidos curriculares; 6) Necesidad de dar respuesta inmediata a la nueva realidad multicultural. Para ello, se necesita una Educación Intercultural desde la igualdad y para la igualdad. 


\section{4 ¿QUÉ RESPUESTAS EN EL PRESENTE Y EN EL FUTURO DEBEN OFRECER LOS SISTEMAS EDUCATIVOS AL ALUMNADO INMIGRANTE PARA MEJORAR SU ATENCIÓN?}

Ante una sociedad cada vez más diversa y en continuo movimiento, los sistemas educativos deben ofrecer una respuesta que sea capaz de integrar toda esa diversidad de manera normalizada, satisfacer las necesidades específicas que, sin duda, se van a derivar de ella y generar la interacción necesaria para que esa heterogeneidad sea fuente de aprendizaje y enriquecimiento mutuo. El Plan Estratégico de Ciudadanía e Integración, que comenzó en 2007-2010 y tuvo una continuación 2011-2014, planteaba una serie de objetivos estratégicos que buscaban precisamente estos fines. En estos años, podemos decir que se han realizado progresos, pero, al mismo tiempo, estamos aún lejos de que se hayan logrado de manera efectiva en su totalidad. Es interesante conocerlos contrastando su excelente planificación con su limitada aplicación práctica.

El primer objetivo se propone "garantizar el acceso del alumnado inmigrante a las etapas de educación obligatoria en igualdad de condiciones". Para favorecer la incorporación del alumnado inmigrante al sistema educativo, es preciso compatibilizar el derecho de las familias a elegir el centro docente sostenido con fondos públicos con la erradicación de la segregación escolar. Asimismo, deben desarrollarse en los centros educativos programas específicos para la acogida, la integración sociocultural y la convivencia escolar y extraescolar.

En segundo lugar, está el reto de "garantizar una educación obligatoria de calidad, independientemente de la condición o procedencia del alumno". Para lograr la igualdad de oportunidades, deben desarrollarse políticas educativas integradoras que reconozcan la presencia del alumnado inmigrante y sus características específicas, ofreciendo los apoyos precisos para responder adecuadamente a las necesidades particulares que presentan ${ }^{2}$.

Resulta crucial "adecuar el sistema educativo a la diversidad del alumnado gestionando adecuadamente dicha diversidad y fomentando la adquisición de conocimientos y competencias interculturales". Ello requiere incluir la perspectiva de la diversidad cultural en la formación inicial y permanente del profesorado, proporcionándole competencias y conocimientos precisos para que pueda responder de la manera más adecuada a los cambios sociales y a un alumnado diverso. Un desarrollo eficiente de la interculturalidad como modelo

\footnotetext{
${ }^{2}$ En este sentido, el Informe Pisa 2006 aboga claramente por la compatibilidad entre la integración y la calidad educativa: La mejora del rendimiento de todos los alumnos y el mantenimiento de los buenos resultados españoles en equidad, procesos compatibles, como demuestra PISA; el trabajo en el entorno educativo de los alumnos para conseguir contrarrestar el efecto de las diferencias culturales, económicas y sociales de los contextos familiares y de los centros; el estímulo y la promoción de la formación docente para que sea más eficaz el trabajo con los alumnos en la adquisición de las competencias básicas. MINISTERIO DE EDUCACIÓN Y CIENCIA. PISA 2006. Programa para la Evaluación Internacional de Alumnos de la OCDE (Informe Español). Madrid: Ministerio de Educación y Ciencia. 2007, p. 102.
} 
de gestión de la diversidad cultural precisa que el sistema educativo se vuelque en el conocimiento, la percepción y la comprensión de grupos y culturas diferentes a los propios, de manera que se logre una convivencia y una interrelación próspera a través de la búsqueda de similitudes y la tolerancia a las diferencias. La incorporación de la educación en valores y para la ciudadanía al currículo es un elemento muy importante en la consecución de tal fin. Por otra parte, resulta igualmente esencial que el sistema educativo facilite y apoye el mantenimiento de la lengua y la cultura de origen de los alumnos inmigrantes ${ }^{3}$.

Para poder incluir al alumnado inmigrante de manera normalizada, es preciso "transformar la escuela en espacio de comunicación, convivencia e integración en el entorno". Las instituciones educativas han de ser espacios integradores de la educación formal e informal. Para ello, resulta esencial llevar a la práctica proyectos educativos integradores en los que participen todos los agentes de la comunidad educativa ${ }^{4}$, promoviendo así desde la escuela la convivencia y la cohesión social y adecuando a la sociedad ante el desafío de la diversidad. En este sentido, destacan actuaciones como las aulas abiertas, los talleres o las tertulias sobre temas que afecten a la comunidad educativa.

Si pretendemos que la educación cumpla con su función de herramienta de progreso personal y social, es necesario "facilitar el acceso del alumnado inmigrante a etapas no obligatorias". La igualdad de oportunidades sólo será efectiva si se promueve el acceso del alumnado inmigrante a las etapas de enseñanza no obligatoria en condiciones de igualdad con el alumnado autóctono. Es especialmente importante flexibilizar el acceso a la educación infantil, dado que una escolarización temprana ayuda en gran medida a la integración en el sistema educativo y proporciona al alumno la base para asimilar los aprendizajes posteriores. También lo es la permanencia en los niveles educativos postobligatorios, que resulta igualmente importante para la completa formación de la persona y condiciona en alto grado la inserción laboral.

En este mismo sentido, también es fundamental "mejorar el acceso de los inmigrantes a la formación de adultos". Para favorecer la inclusión de las personas inmigrantes en la formación continua, se precisa que ésta adapte su oferta a las necesidades y particularidades específicas del alumnado y que se promueva la formación de adultos como una vía

\footnotetext{
${ }^{3}$ El Dictamen del Foro para la Integración Social de los Inmigrantes también avala este aspecto. Pide un esfuerzo para conservar la lengua y los valores culturales de origen del alumnado inmigrante y que la comunidad educativa asuma un papel activo en la defensa de la diversidad cultural. En: FORO PARA LA INTEGRACIÓN SOCIAL DE LOS INMIGRANTES. Dictamen del Foro para la Integración Social de los Inmigrantes sobre el Proyecto de Plan Estratégico de Ciudadanía e Integración 2007-2010. Madrid: Ministerio de Trabajo y Asuntos Sociales. FISI/2007/D1. 2007.

${ }^{4}$ En este sentido, el Dictamen del Foro para la Integración Social de los Inmigrantes se detiene a proponer cuestiones como institucionalizar y estimular las redes de colaboración entre los profesores, los centros, los recursos y las instituciones que trabajan con inmigrantes; aumentar los medios materiales y humanos; y fomentar la transferencia de buenas prácticas, a fin de garantizar una educación de calidad y para todos. En: Ibidem. p. 7.
} 
fundamental para la inserción y la promoción laboral. Para ello, se necesita también la implicación de los agentes sociales.

En muchas ocasiones, las trabas burocráticas y la rigidez administrativa es un obstáculo difícil de salvar. Es preciso "mejorar los procedimientos de homologación de las titulaciones académicas". Se necesita un mayor conocimiento y una mayor agilización de los procedimientos de homologación de las titulaciones académicas para que las personas inmigrantes puedan recorrer itinerarios profesionales que se correspondan con su nivel formativo.

Si analizamos los datos de la Encuesta Nacional de Inmigrantes 2007 del Instituto Nacional de Estadística ${ }^{5}$, vemos cómo el 97,5\% de los hijos de extranjeros de entre 4 y 16 años, periodo que comprende toda la enseñanza obligatoria, que comienza a los 6 años, se encuentran escolarizados. Con respecto al conocimiento de la lengua de referencia, una de las dificultades más comunes en la adaptación a un nuevo sistema educativo, el 91,6\% afirma hablar bien $(20,4 \%)$ o muy bien el castellano $(71,2 \%)$. Es más, si se valora el nivel de conocimiento del castellano entre los chicos mayores de 16 años, crece hasta el 92,3\% el porcentaje que afirma hablar bien $(15,5 \%)$ o muy bien $(76,8 \%)$ el castellano.

Imagen 1. Escolarización en enseñanzas obligatorias.

\section{Escolarización en enseñanzas obligatorias de los hijos de inmigrantes}

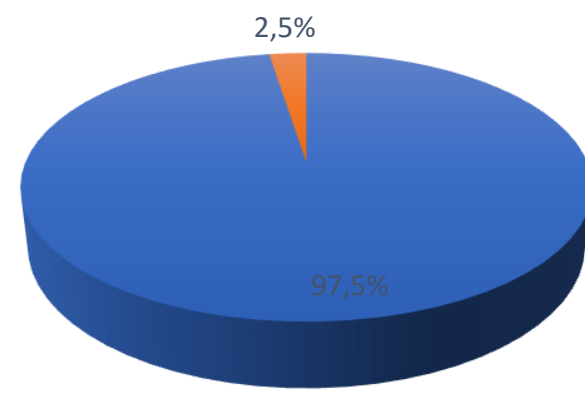

Escolarizados No escolarizados

Fuente: Instituto Nacional de Estadística. Encuesta Nacional de Inmigrantes 2007.

\footnotetext{
${ }^{5}$ La exhaustiva Encuesta Nacional de Inmigrantes, valiosísima fuente de datos para la investigación de las migraciones en panorámica interdisciplinar, fue publicada por el Instituto Nacional de Estadística en 2009, pero tristemente no se ha vuelto a reeditar hasta la fecha. Instituto Nacional de Estadística (2009): Encuesta Nacional de Inmigrantes 2007: una monografía. Madrid. Op. Cit.
} 
En cuanto a la escolarización postobligatoria, el 30,9\% de los hijos de los nacidos en el extranjero continúa escolarizado después de cumplir los 17 años. La lectura de los datos nos indica que, en la educación postobligatoria, fundamental para lograr el ascenso social, queda aún mucho camino por recorrer. Al igual que ocurre con la población autóctona, la influencia del entorno familiar resulta determinante. Muestra de ello es que el porcentaje de escolarización de los hijos de inmigrantes de 17 o más años asciende a un 72,9\% en el caso de que sus padres posean un nivel alto de cualificación. Ello reafirma la idea de que la promoción de la educación postobligatoria entre los inmigrantes requiere medidas de orientación y sensibilización con las familias, que generalmente resultan determinantes en la decisión de sus hijos de continuar o no con los estudios postobligatorios.

Imagen 2. Escolarización en Enseñanzas Postobligatorias.

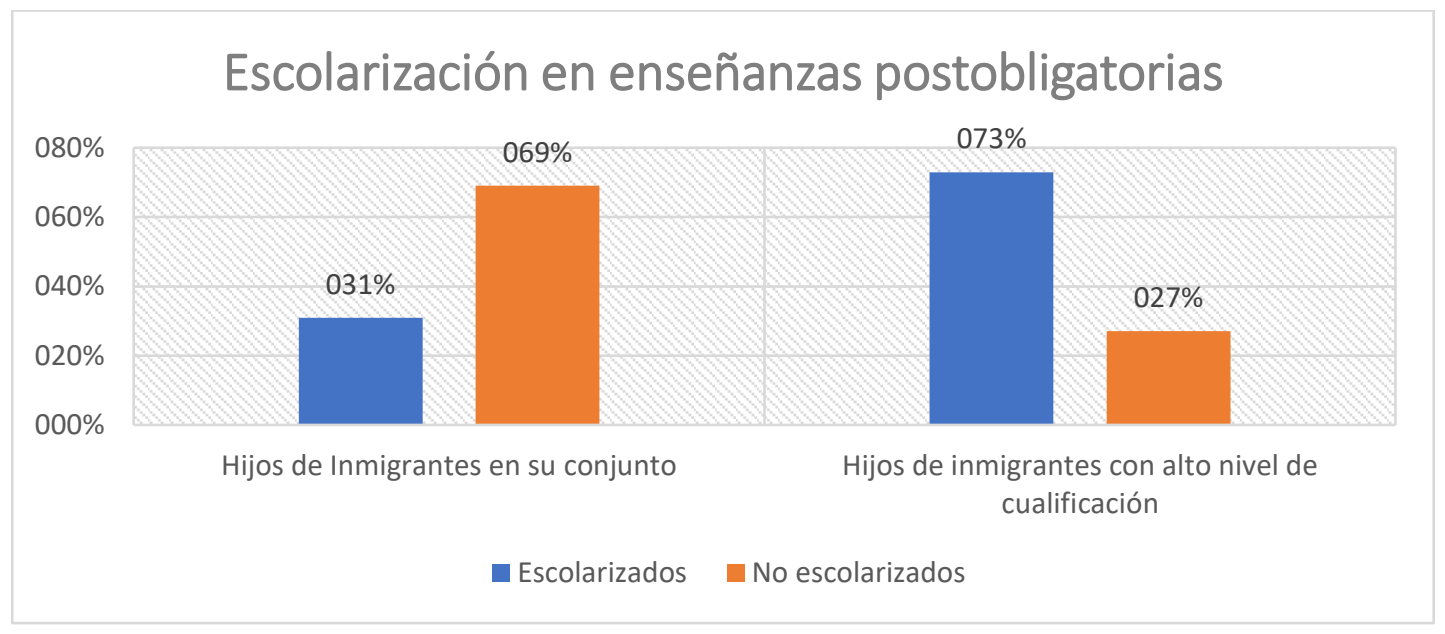

Fuente: Instituto Nacional de Estadística. Encuesta Nacional de Inmigrantes 2007.

En los años consiguientes, la cifra de alumnos extranjeros matriculados en las enseñanzas no universitarias aumentó hasta en más de un punto porcentual, llegando en el curso 2008-2009 al porcentaje de 9,8\%, se mantiene por encima de los nueve puntos porcentuales hasta el curso 2012-2013 y comienza a bajar ligeramente a partir de ahí, situándose en el curso 2017-2018 en el 8,8\%. 
Imagen 3. Evolución alumnado extranjero.

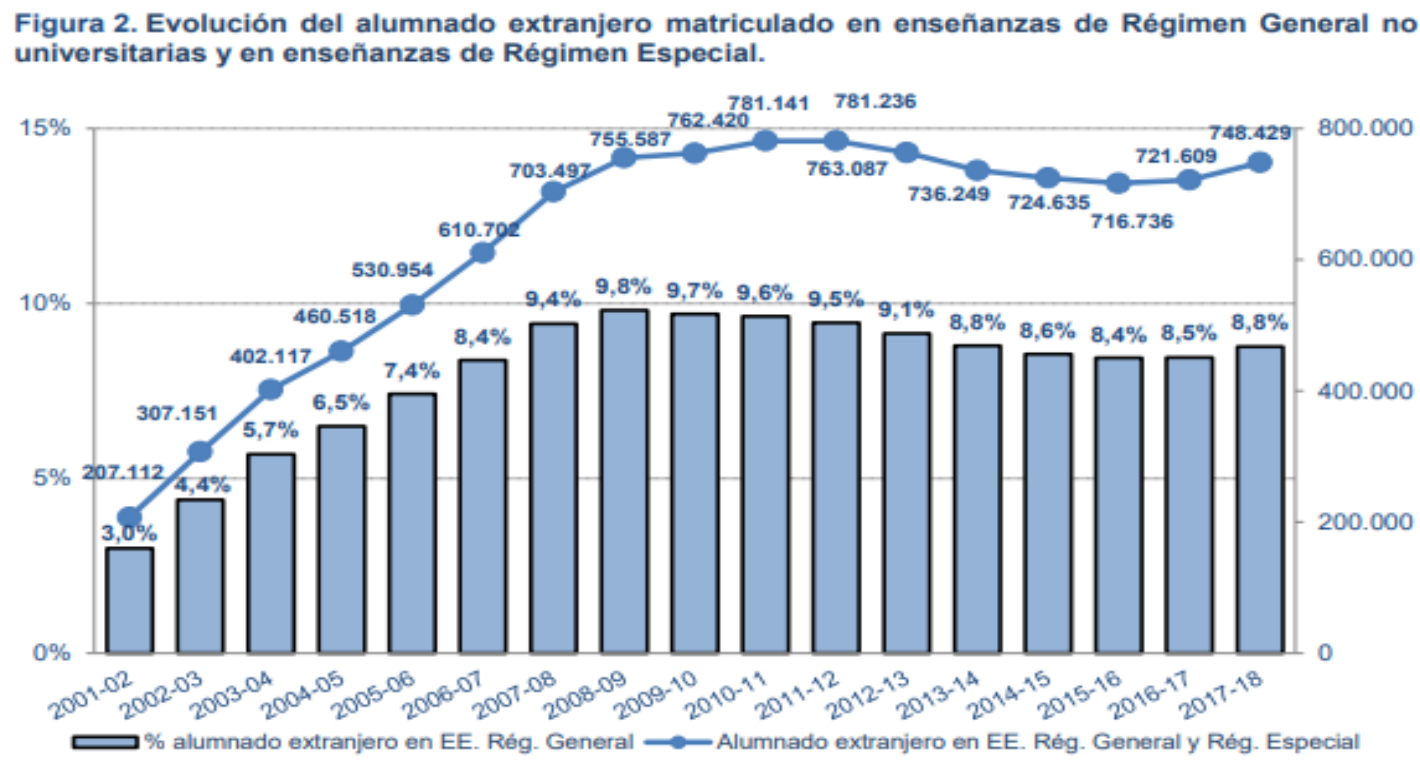

Fuente: Ministerio de Educación y Formación Profesional. Estadística de Enseñanzas No Universitarias: Datos Avance 2017-2018.

También son necesarias medidas de integración a todos los niveles. En este sentido, querría apelar a la encomiable labor que realizan los mediadores interculturales. Es necesaria una mayor colaboración entre el ámbito educativo y los servicios sociales con capacidad para promover conjuntamente la interacción intercultural dentro y fuera de la escuela. La interacción entre culturas es la base de la integración y también es el elemento esencial de la interculturalidad. El artículo 4.1. de la Convención sobre la Protección y la Promoción de la Diversidad de las Expresiones Culturales (aprobada en 2005 y ratificada en 2007) define la interculturalidad así: "La 'interculturalidad' se refiere a la presencia e interacción equitativa de diversas culturas y la posibilidad de generar expresiones culturales compartidas, adquiridas por medio del diálogo y de una actitud de respeto mutuo". Sería muy sano y recomendable que no perdiéramos de vista esta referencia. Por ahí está el camino.

\section{REFERENCIAS}

ARROYO GONZÁLEZ, María José. La educación intercultural: un camino hacia la inclusión educativa. Revista de Educación Inclusiva, v.6, n.2, p. 144-159, junio de 2013.

COMISIÓN EUROPEA. Estrategia marco contra la discriminación y por la igualdad de oportunidades para todos. Comunicación de la Comisión Europea al Consejo, al Parlamento Europeo, al Comité Económico y Social Europeo y al Comité de las Regiones. COM (2005) 224 final. Bruselas, 1 de junio de 2005. 
COMISIÓN EUROPEA. Programa de La Haya: diez prioridades para los próximos cinco años; una asociación para la renovación europea en el ámbito de la libertad, la seguridad y la justicia. Comunicación de la Comisión Europea al Consejo y al Parlamento Europeo. COM (2005) 184 final. Bruselas, 10 de mayo de 2005.

COMISIÓN EUROPEA. Justicia, libertad y seguridad en Europa desde 2005: una evaluación del Programa de la Haya y del Plan de Acción. Comunicación de la Comisión al Consejo, al Parlamento Europeo, al Comité Económico y Social Europeo y al Comité de las Regiones. COM (2009) 263 final. Bruselas, 10 de junio de 2009.

CONFERENCIA GENERAL DE LA ORGANIZACIÓN DE LAS NACIONES UNIDAS PARA LA EDUCACIÓN, LA CIENCIA Y LA CULTURA. Convención sobre la Protección y la Promoción de la Diversidad de las Expresiones Culturales. París: UNESCO, 20 de octubre de 2005.

ESCARBAJAL FRUTOS, A. La educación intercultural en los centros educativos. Revista Electrónica Interuniversitaria de Formación del Profesorado, v.17, n.2, p. 29-43.

FERNÁNDEZ SIERRA, J. Alumnado inmigrante en la ESO: vulnerabilidad pedagógica del sistema educativo. Educación XX1, v.20, n.1, p. 121-140, 2017.

FORO PARA LA INTEGRACIÓN SOCIAL DE LOS INMIGRANTES. Dictamen del Foro para la Integración Social de los Inmigrantes sobre el Proyecto de Plan Estratégico de Ciudadanía e Integración 2007-2010. Madrid: Ministerio de Trabajo y Asuntos Sociales. FISI/2007/D1. 2007.

FORO PARA LA INTEGRACIÓN SOCIAL DE LOS INMIGRANTES. Informe sobre la situación de la Integración Social de los Inmigrantes y Refugiados en 2009. Madrid: Monografías del Foro para la Integración Social de los Inmigrantes, Ministerio de Trabajo e Inmigración. 2009.

FORO PARA LA INTEGRACIÓN SOCIAL DE LOS INMIGRANTES. La integración del alumnado inmigrante en la escuela. Madrid: Monografías del Foro para la Integración Social de los Inmigrantes. Ministerio de Trabajo e Inmigración. 2009.

GARRIDO RODRÍGUEZ, Pedro. El panorama internacional ante la educación y la pobreza. Revista sobre educación y liderazgo educativo, n.o 5, Ed. Fórum Europeo de Administradores de la Educación. p. 11-14, 2020.

GARRIDO RODRÍGUEZ, Pedro. Inmigración y diversidad cultural en España: su gestión desde la bonanza económica a la crisis. Madrid: Editorial Fundamentos, 2014.

GIMÉNEZ GÁMEZ, Rafael; GOENECHEA PERMISÁN, Cristina. Educación para una ciudadanía intercultural. Madrid: Editorial Síntesis, 2014.

INSTITUTO NACIONAL DE ESTADÍSTICA. Encuesta Nacional de Inmigrantes 2007: una monografía. Madrid: INE, 2009.

JEFATURA DEL ESTADO. Ley Orgánica 2/2006, de 3 de mayo, de Educación. BOE, no.106, de jueves 4 de mayo de 2006. 
JEFATURA DEL ESTADO. Ley Orgánica 2/2009, de 11 de diciembre, de reforma de la Ley Orgánica 4/2000, de 11 de enero, sobre derechos y libertades de los extranjeros en España y su integración social. BOE, n.․29 299, de 12 de diciembre de 2009.

JEFATURA DEL ESTADO. Ley Orgánica 8/2013, de 9 de diciembre, para la mejora de la calidad educativa. BOE, n.․․ 295, de 10 de diciembre de 2013.

LEIVA, Juan. Interculturalidad y estilos de aprendizaje: nuevas perspectivas pedagógicas. International Journal of Educational Research and Innovation (IJERI), 3, p. 36-51, 2015.

MARTínEZ USARRALDE, María Jesús; LLORET CATALÁ, María del Carmen; CÉSPEDES RICO, Manuel. Lo que hacen las mejores escuelas integradoras de alumnado inmigrante: indicadores de buenas prácticas. Pedagogía social: revista interuniversitaria, $n .029$, p. 4154, 2017.

MINISTERIO DE EDUCACIÓN Y CIENCIA. PISA 2006. Programa para la Evaluación Internacional de Alumnos de la OCDE (Informe Español). Madrid: Ministerio de Educación y Ciencia, 2007.

MINISTERIO DE EDUCACIÓN Y FORMACIÓN PROFESIONAL. Estadística de enseñanzas no universitarias: datos avance 2017-2018. Madrid: Ministerio de Educación y Formación Profesional, 2019.

MINISTERIO DE TRABAJO Y ASUNTOS SOCIALES. Plan Estratégico Ciudadanía e Integración (2007-2010). Madrid: Ministerio de Trabajo y Asuntos Sociales, 2007.

PEGALAJAR PALOMINO, Ma del Carmen; COLMENERO RUIZ, Mạ Jesús. Inteligencia emocional en alumnado de Educación Secundaria en contextos multiculturales. Electronic Journal of Research in Educational Psychology, 12(2), n. 33, p. 325-342, 2014.

PÉREZ ESPARRELS, María del Carmen; RAHONA LÓPEZ, Marta. La inmigración en el sistema educativo español y sus implicaciones para la política educativa. In: CACHÓN RODRÍGUEZ, Roberto; LAPARRA NAVARRO, Miguel. Inmigración y políticas sociales. Barcelona: Ed. Bellaterra, 2009. p. 149-180.

SECRETARÍA GENERAL DE POLÍTICAS DE IGUALDAD. Plan Estratégico de Igualdad de Oportunidades (2008-2011). Madrid: Ministerio de Trabajo y Asuntos Sociales, 2007.

TORRES, Francisco; GADEA, Mạ. Elena. Crisis, inmigración y sociedad. Madrid: Talasa; Ed. Ágora, 2015.

Revisión gramatical: Karine Taiza Mendes

Correo electrónico: Karine ktm@hotmail.com 